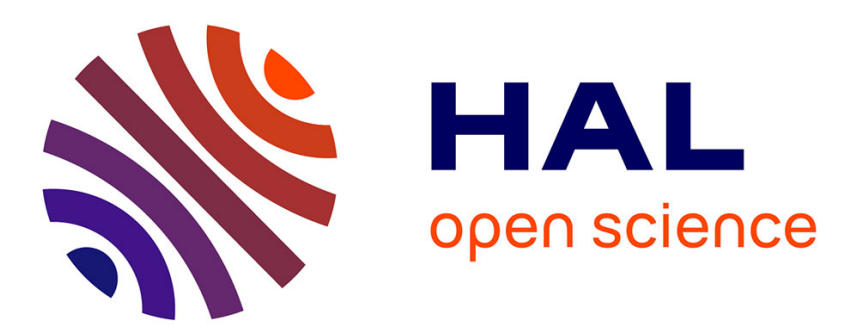

\title{
Petite agriculture familiale et agriculture d'entreprise en Argentine : politiques d'encadrement et rationalités antagonistes
}

Marie Gisclard, Martine Guibert

\section{- To cite this version:}

Marie Gisclard, Martine Guibert. Petite agriculture familiale et agriculture d'entreprise en Argentine : politiques d'encadrement et rationalités antagonistes. Problèmes d'Amérique Latine, 2017, 104 (1), pp.101-119. 10.3917/pal.104.0101 . hal-02081950

\section{HAL Id: hal-02081950 \\ https://hal-univ-tlse2.archives-ouvertes.fr/hal-02081950}

Submitted on 2 Apr 2019

HAL is a multi-disciplinary open access archive for the deposit and dissemination of scientific research documents, whether they are published or not. The documents may come from teaching and research institutions in France or abroad, or from public or private research centers.
L'archive ouverte pluridisciplinaire HAL, est destinée au dépôt et à la diffusion de documents scientifiques de niveau recherche, publiés ou non, émanant des établissements d'enseignement et de recherche français ou étrangers, des laboratoires publics ou privés. 


\section{PETITE AGRICULTURE FAMILIALE ET AGRICULTURE D'ENTREPRISE EN ARGENTINE : POLITIQUES D'ENCADREMENT ET RATIONALITÉS ANTAGONISTES}

Marie Gisclard, Martine Guibert

ESKA | « Problèmes d'Amérique latine »

2017/1 N 104 | pages 101 à 119

ISSN 0765-1333

ISBN 9782747226820

Article disponible en ligne à l'adresse :

http://www.cairn.info/revue-problemes-d-amerique-latine-2017-1-page-101.htm

\section{Pour citer cet article :}

Marie Gisclard, Martine Guibert« Petite agriculture familiale et agriculture d'entreprise en Argentine : politiques d'encadrement et rationalités antagonistes », Problèmes d'Amérique latine 2017/1 ( $\left.\mathrm{N}^{\circ} 104\right)$, p. 101-119.

DOI 10.3917/pal.104.0101

Distribution électronique Cairn.info pour ESKA.

(C) ESKA. Tous droits réservés pour tous pays.

La reproduction ou représentation de cet article, notamment par photocopie, n'est autorisée que dans les limites des conditions générales d'utilisation du site ou, le cas échéant, des conditions générales de la licence souscrite par votre établissement. Toute autre reproduction ou représentation, en tout ou partie, sous quelque forme et de quelque manière que ce soit, est interdite sauf accord préalable et écrit de l'éditeur, en dehors des cas prévus par la législation en vigueur en France. Il est précisé que son stockage dans une base de données est également interdit. 
VARIA 


\title{
Petite Agriculture familiale ET AGRICULTURE D'ENTREPRISE EN ARGENTINE : POLITIQUES D'ENCADREMENT ET RATIONALITÉS ANTAGONISTES
}

\author{
Marie GISCLARD* \& Martine GUIBERT**
}

\section{INTRODUCTION}

Le secteur agricole argentin réunit des modèles pluriels de production présentant des formes différenciées de relations au politique, à l'économique, à la société et au territoire. Généralement, la différenciation entre ces modèles est décrite selon un mode binaire entre, d'une part, une agriculture de base entrepreneuriale, capitalisée et tournée vers les marchés internationaux, et, d'autre part, une petite agriculture de base familiale, reposant sur une logique de subsistance et/ou d'approvisionnement des marchés locaux et nationaux. Elle occulte toutefois une réalité plus complexe qui articule les modèles tout autant qu'elle les oppose. Des grands consortiums financiers aux communautés indigènes, de l'agriculture familiale innovante aux petits producteurs fragiles, les formes d'organisation sociale et économique en agriculture se sont considérablement diversifiées en Argentine, comme dans d'autres contextes géographiques, voisins ou plus lointains.

* Labex DynamiTe, portant la référence ANR-11-LABX-0046, membre de PRODIG. marie.gisclard59@gmail.com

** Université Toulouse - Jean Jaurès, Département de géographie-aménagementenvironnement, UMR CNRS 5193 LISST/Dynamiques rurales. guibert@univ-tlse2.fr 
Au cœur de la reprise macro-économique de l'Argentine des années 2000, après la crise historique de 1998-2001, l'agriculture de ce pays est présentée dans la littérature scientifique comme un secteur animé par la révolution des biotechnologies et par des conditions financières et commerciales attractives, et intégré dans des chaînes de valeur globales dont les normes reflètent des standards internationaux ${ }^{1}$. Or, c'est dans ce contexte qui souligne les résultats macro-productifs (augmentation de la production et de la productivité) du secteur agricole, et sa contribution à la balance commerciale et aux recettes fiscales, que le gouvernement de Cristina Kirchner (deux mandats 2007-2011 et 2011-2015) a créé, à la fin des années 2000, un secrétariat d'État à la petite agriculture familiale ${ }^{2}$ qui est justement exclue de cette dynamique exportatrice.

Au moyen d'une réflexion qui repose sur des travaux de terrain menés ces dix dernières années (principalement dans les régions de la Pampa et du Chaco), d'entretiens semi-directifs, de la consultation des publications des organisations professionnelles et politiques, nous proposons une interprétation des ressorts de la coexistence de ces modèles de développement agricole a priori antagonistes. Nous puisons dans le temps long (depuis la modernisation de l'agriculture des années 1960) des éléments de mise en perspective de leurs trajectoires socio-économiques et des mécanismes de leur légitimation politique, et nous cherchons les points d'inflexion qui ont conduit à leur bifurcation. De plus, pour comprendre les trajectoires de ces modèles productifs et les rationalités dont ils relèvent, nous nous concentrons, non pas sur les techniques de production qui ont souvent été au cœur de la description de ces modèles, mais sur les techniques d'encadrement ${ }^{3}$ dont ils ont fait l'objet. La notion de technique d'encadrement décrit les dimensions culturelles et politiques des interactions entre les hommes qui régissent une société et contribuent à son efficience sociale et écologique ${ }^{4}$. Elle est une façon de considérer la relation qu'entretiennent les hommes avec la nature. Elle rend ainsi compte d'une rationalités, c'est-à-dire de l'organisation de certains moyens par rapport à une fin, dominante à un moment donné. Dans notre cas, nous observons et nous identifions ces techniques d'encadrement et ces rationalités politiques et économiques à partir des cadres de régulation mis successivement en place par l'État.

${ }^{1}$ D. Requier-Desjardins, M. Guibert, E. A. Bühler, « La diversité des formes d'agricultures d'entreprise au prisme des réalités sud-américaines ", Économie rurale, $\mathrm{n}^{\circ}$ 344, pp. 45-60, 2014.

${ }^{2}$ M. Gisclard, G. Allaire, "L'institutionnalisation de l'agriculture familiale en Argentine : vers la reformulation d'un référentiel de développement rural », Autrepart, n 62, pp. 201-216, 2012.

${ }^{3}$ P. Gourou, Pour une géographie humaine, Paris, Flammarion, 1973.

${ }^{4}$ C. Barnaud, Équité, jeux de pouvoir et légitimité : les dilemmes d'une gestion concertée des ressources renouvelables. Mise à l'épreuve d'une posture d'accompagnement critique dans deux systèmes agraires des hautes terres du Nord de la Thaïlande. Thèse de doctorat en géographie, Paris X Nanterre, 2008.

${ }^{5}$ M. Santos, La nature de l'espace, Paris, L’Harmattan, 1997. 
Dans une première partie, nous relatons comment l'État argentin a privilégié dans les années 1970 une technique d'encadrement qui a promu une agriculture entrepreneuriale et exportatrice qui a été au cœur du développement de la production de la région pampéenne. Nous montrons ensuite à travers le cas de la région du Chaco comment, à cette même période, des acteurs prennent en charge les exclus de l'intervention de l'État, et qui sont la base politique et culturelle de la petite agriculture familiale actuelle. Dans la deuxième partie, nous voyons comment l'orientation néolibérale des années 1990 provoque un revirement du rôle de l'État dans les techniques d'encadrement de chaque modèle. Dans la troisième partie, nous constatons qu'une reconnaissance politique des deux modes d'agriculture caractérise les années 2000 , et ce dans un contexte de cohabitation conflictuelle ou, parfois, d'hybridation entre les différentes formes d'organisation de la production, ce qui oblige à ne pas adopter une lecture trop duale de la réalité.

\section{RATIONALITÉ TECHNIQUE ET ÉCONOMIQUE VERSUS CONTRE-RATIONALITÉ SOCIALE ET POLITIQUE}

Á partir des années 1960, l'État argentin encadre un processus de modernisation qui constitue une technique d'encadrement basée sur une rationalité essentiellement technique et économique, et qui va surtout bénéficier à la Pampa humide. Sur ses marges, dans le Chaco, les exclus du changement vont proposer une autre technique d'encadrement reposant sur une autre rationalité, davantage sociale et politique.

\section{Le soutien aux exploitations pampéennes et aux "producteurs dyna- miques"}

Le processus de modernisation s'enclenche en Argentine à partir du milieu des années 1950. Il s'inscrit dans la stratégie macro-économique d'industrialisation par substitution des importations que suivent les pays latino-américains et dans laquelle le secteur agricole joue un rôle important : la création de devises à l'exportation doit permettre de financer le développement du secteur industriel. Or, l'agriculture argentine est dominée par de grands propriétaires terriens qui entretiennent des liens forts avec le pouvoir politique. Ils pratiquent l'élevage extensif et valorisent une rente sur les marchés internationaux, sans chercher à moderniser leurs domaines, souvent peu rentables. Le protectionnisme en vigueur aggrave le manque d'investissements et d'adoption d'innovations technologiques, occasionnant un certain retard par rapport au processus de croissance agricole des États-Unis ou d'autres pays agro-exportateurs (Australie, Canada).

La modernisation vise à capter la demande européenne en hausse après la seconde guerre mondiale (viande, céréales et, très vite, protéine de soja). Comme dans d'autres régions du monde, elle conduit à la sectorisation et à la professionnalisation de l'agriculture, et elle se base sur deux dimen- 
sions : sociale et productive. L'une cherche à stabiliser une base sociale de producteurs dynamiques, grands et moyens, de type "farmers », capables d'augmenter les rendements et qui seront les cibles de l'action de l'État ${ }^{6}$. $\mathrm{La}$ "déconcentration » des estancias d'élevage et l'accès à la terre pour les métayers se feront sans trop nuire aux intérêts de l'aristocratie terrienne. L'autre est la moto-mécanisation de la production et la mise à disposition d'innovations techniques. Dans cet objectif, l'INTA (Instituto Nacional de Tecnología Agropecuaria), créé en 1956, est chargé de combler la brèche technologique qui sépare la Pampa des autres grandes régions agricoles du monde. Il encourage la mécanisation et l'usage de semences hybrides, de pesticides et de génétique animale, à travers des agences de vulgarisation (extensión) implantées dans tout le pays. De manière à favoriser l'adoption de ces technologies par les agriculteurs, l'État facilite l'accès au crédit et améliore les infrastructures rurales : électrification, chemins, voies ferrées. Le tracteur et la moissonneuse se généralisent. La commercialisation est régulée depuis les années 1930 à travers des organismes de contrôle (Junta Nacional de carnes, Junta Nacional de granos, etc.) ; tout comme l'industrialisation, elle est promue par l'IAPI (Instituto Argentino de Promoción Industrial) avec le gouvernement de Perón des années 1940. Les coopératives s'imposent au cœur des filières.

Néanmoins, le processus de modernisation est incomplet : il est axé sur la rationalisation de la production et son adaptation aux marchés internationaux, et non sur le développement et l'amélioration des conditions de vie et de l'accès aux services dans les espaces ruraux. La politique de l'État "n'avait pas pour intention de réaliser un quelconque changement social ou politique d'envergure. Alors que le pays transformait sa structure productive à travers la croissance forcée de son industrie, [...] une intense urbanisation et des migrations rurales vers les villes, rien de tout cela ne s'est vu accompagné par des politiques sociales, ni par un projet clair d'industrialisation, et l'oligarchie gouvernait selon un mode féodal, à travers une démocratie fictive [...] et la répression ${ }^{7}$. Par ailleurs, le processus de modernisation se cristallise dans la Pampa humide, centre politique et économique du pays, et qui réunit les meilleures terres à grains du pays. Région bordée par le $\mathrm{Pa}$ raná, elle bénéficie de la proximité de terminaux portuaires modernes et elle symbolise, de fait, l'agriculture argentine exportatrice.

\section{Les régions extra-pampéennes au défi de rationalités exogènes : le cas du Chaco}

Les régions extra-pampéennes, dites "régions périphériques », ont été spécialisées dans des productions orientées vers la couverture des besoins nationaux. Ainsi, le Chaco, au nord-ouest du pays, est tourné depuis les an-

${ }^{6}$ O. Barsky, J. Gelman, Historia del agro Argentino, Buenos Aires, Ed. Grijalbo, 2001.

${ }^{7}$ M. Rapoport, "El enigma argentino », 21.11.2010, Página/12, <http://www. pagina12.com.ar/diario/suplementos/cash/17-477> 
nées 1920 vers la production de coton. Le processus de modernisation conduit à une mécanisation similaire à celle en cours dans la Pampa. Les rendements augmentent, ce qui permet de compenser la diminution des revenus lors des épisodes fréquents de baisse des prix du coton. La filière est en effet dépendante du soutien de l'État. Mais, suite à la suppression dans les années 1960 de la Junta nacional de algodón et à la fin des prix garantis, le marché international va devenir le principal régulateur. Les producteurs doivent s'adapter, les structures d'exploitation sont agrandies et la déforestation s'accélère pour incorporer de nouvelles terres : il n'y a pas de parcelles qui ne soient pas consacrées à la production de coton.

Un processus d'exode rural long et continu démarre ; il s'explique par l'expulsion des producteurs marginalisés et par l'accentuation des différences de qualité de vie entre ville et campagne. La modernisation technique n'est, en effet, pas accompagnée par une réelle amélioration des conditions de vie en milieu rural. L'isolement des fermes, la précarité des chemins de terre, et l'absence d'eau courante et, dans certains cas, de l'électricité, incitent le chef de famille à installer sa famille en ville où elle accède aux services publics. Après être resté toute la semaine sur l'exploitation, celui-ci regagne à son tour le centre urbain, grâce à la généralisation de l'automobile.

Dès lors, seules les exploitations grandes et moyennes accèdent aux innovations techniques. Les plus petits producteurs, qui cultivent moins de 20 ha et représentent dans les années 1970 plus de $84 \%$ des exploitations de la province du Chaco, n'ont pas les revenus suffisants pour opérer les changements nécessaires. $60 \%$ d'entre eux n'ont pas accès au crédit en raison de l'absence de titre de propriété ${ }^{8}$. Les producteurs familiaux trop endettés et qui ne peuvent pas s'agrandir sont expulsés.

Ainsi, les pouvoirs publics favorisent explicitement l'hégémonie d'agriculteurs familiaux moyens, dynamiques et compétitifs, et la consolidation d'entreprises agricoles : "[...] Notre producteur se structure à la manière des farmers et producteurs moyens du Canada et des États-Unis ; propriétaires hautement mécanisés, technicisés, réalisant des cultures intensives et regroupés pour la commercialisation de leurs productions " (ministre de l'économie de la province du Chaco en $1978^{9}$ ). Ils ne feront rien pour enrayer la fracture entre les différentes strates productives et la concentration foncière. Celle-ci découle de l'achat de terres aux producteurs qui ne peuvent plus poursuivre leur activité, ou bien de la mise en culture de nouveaux lots défrichés aux dépens de la forêt. L'Institut de Colonisation du Chaco, organisme de l'État provincial chargé d'administrer les terres publiques, ne se donne pas comme priorité de régulariser la situation des populations qui y sont installées de longue date, sans titre de propriété. Il devient même, au cours du temps, l'un des instruments de la corruption

${ }^{8}$ E. Bruniard, « El Gran Chaco Argentino. Ensayo de interpretación geográfica », Geográfica. Revista del instituto de geografía, Resistencia, UNNE, n 4, 1978.

${ }_{9}$ Cité dans : J.P. Roze, Lucha de clase en el Chaco contemporáneo, Resistencia, Librería de la Paz, 2007, p. 163. 
provinciale. Ces années de privatisation des terres publiques, en réserve depuis la conquête du Chaco, bénéficient aux producteurs familiaux moyens et aux entreprises agricoles, cibles de la rationalité technique et économique qui prévaut.

Avec la junte militaire au pouvoir à partir de 1976, la libéralisation de l'économie prend le relais de l'expérience protectionniste. Un régime d'accumulation tiré par les exportations est privilégié ${ }^{10}$. L'INTA, jusque-là empreint d'un paradigme " éducatif » orienté vers « la famille agricole », prône désormais le transfert de technologies vers " l'exploitation compétitive »"

Dans le Chaco, les producteurs grands et moyens doivent faire face à la perte de rentabilité du coton. Ils se diversifient en se lançant dans des productions d'origine tempérée (tournesol, maïs, sorgho, élevage bovin). Cette substitution, désignée par le terme de "pampéanisation ", est décrite de manière critique, comme une façon de " penser et agir comme si les paquets technologiques et les types d'usage des sols étaient interchangeables entre des écorégions très différentes ${ }^{12}$. Elle produit une transformation des structures sociales et économiques, et des conditions environnementales régionales.

\section{L'émergence de "contre-rationalités ": les origines du développement rural en Argentine}

Le processus de modernisation s'apparente à un pacte moderniste (Allaire, 2002) axé sur la transformation des structures productives via l'investissement. Il exclut certaines catégories et délaisse les dimensions sociales et culturelles. Si les producteurs pampéens sont encadrés par plusieurs organismes (État, recherche publique, syndicats, groupements de producteurs), les autres acteurs du monde rural vivent une marginalisation croissante. Dans la région du Chaco, les petits producteurs et les populations pauvres (indigènes, ouvriers agricoles) ont été exclus du pacte entre secteur agricole et État. Ils n'intègrent pas la « profession » agricole. Peu affiliés aux coopératives (qui perdent de leur prépondérance), ils restent dépendants des commerçants et intermédiaires locaux. Ils entretiennent des relations clientélistes avec les élites politiques locales et ne sont pas représentés par les principaux syndicats agricoles. Avec la crise du coton, ils rejoignent les centres urbains mais sont difficilement absorbés par une in-

${ }^{10}$ R. Boyer, J.C. Neffa, « La crise argentine 1976-2001 : lectures institutionnalistes et régulationnistes », working paper $n^{\circ}$ 46, Paris School of economics, 2007.

${ }^{11}$ C. Alemany, "Apuntes para la construcción de los períodos históricos de la Extensión Rural del INTA », dans collectif, La Extensión Rural en debate: concepciones, retrospectivas, cambios y estrategias para el Mercosur, Buenos Aires, Ed. INTA, 2003, pp. 137-171.

${ }^{12}$ J. Morello, W. Pengue, Y. Rodríguez, « Etapas de uso de los recursos y desmantelamiento de la bioma del Chaco ", dans A. Brown, U. Martinez Ortiz, M. Acerbi, J. Corcuera (eds.) La situación ambiental argentina 2005, Buenos Aires, Fundación Vida Silvestre Argentina, 2006. 
dustrie déclinante, ou bien ils se maintiennent mais, dans ce cas, hors des cadres d'intervention établis par l'État.

Ces populations rurales pauvres peuvent être considérées comme des « contre-rationalités » au sens de Santos ${ }^{13}$, c'est-à-dire des acteurs qui répondent à d'autres logiques que la rationalité dominante. Elles vont alors être l'objet d'une autre technique d'encadrement que celle mise en place par l'État et qui prend deux orientations : d'une part, le renforcement de la dimension sociale et culturelle de la modernisation agricole, et, de l'autre, des actions en faveur des exclus. Cette technique est construite par des acteurs porteurs d'une vision plus large du développement agricole qui vont construire des organisations telles que :

\section{* Le Movimiento Rural de Acción Católica (MR)}

Á l'échelle du pays, le MR se consolide entre 1958 et 1972 comme un mouvement de "promotion humaine intégrale ». Il cherche à organiser la représentation politique et professionnelle des agriculteurs pour qu'ils participent aux transformations impulsées par l'État. Inspiré de la Théologie de la Libération, il propose aux petits producteurs éducation et émancipation du patron ou du caudillo ${ }^{14}$ local. Il s'adresse aussi aux entrepreneurs agricoles et aux estancieros, acteurs en lien avec le pouvoir politique et économique, et donc les plus à même d'engager un changement. Enfin, le MR accompagne l'INTA dans son travail local et adapte, à la réalité des producteurs familiaux du Chaco, le fonctionnement des CREA (groupes de vulgarisation construits sur le modèle des CETA français ${ }^{15}$ associés, dans la Pampa, à la bourgeoisie rurale.

Dans le Chaco, en pleine crise du coton au début des années 1970, le MR donne naissance aux Ligas Agrarias, mouvement syndical revendicatif qui dénonce la concentration des ressources autour d'un modèle productif qui bénéficie aux entreprises et qui accentue la marginalisation des producteurs familiaux et des ouvriers agricoles ${ }^{16}$. " Avec l'apparition des Ligas Agrarias dans l'histoire du pays en 1971, il semblait que les Argentins, nous découvrions enfin "nos paysans". C'était comme se réveiller d'un mauvais rêve dans lequel les paysans n'existaient pas comme une force sociale significative ${ }^{17}$. Mais l'action des Ligas Agrarias restera inaboutie : le régime

${ }^{13}$ M. Santos, La nature de l'espace, Paris, L'Harmattan, 1997.

${ }^{14}$ Leader politique, local ou national, qui entretient une relation autoritaire ou paternaliste avec les masses populaires qui le suivent, et qui incarne les valeurs identitaires et culturelles d'une région ou d'un pays.

${ }^{15}$ Centres d'Etudes Techniques Agricoles créés en 1944.

${ }^{16}$ Des mouvements similaires au MR se retrouvent à cette époque dans de nombreux pays d'Amérique latine, notamment au Brésil (avec les ligues agraires dans le Nord) où le christianisme de libération a inspiré le mouvement actuel des Sans-Terre.

${ }^{17}$ E. Archetti, «Ideología y organización sindical: las Ligas Agrarias del norte de Santa Fe », Desarrollo Económico, n 111, 1998, pp. 447-461. (traduction par nos soins). 
dictatorial qui s'empare du pouvoir en 1976 va poursuivre ses membres et décimer le mouvement.

\section{* Les EFA et les ONG}

Le MR crée également les Écoles de la Famille Agricole (EFA), inspirées des maisons familiales rurales françaises, qui visent à résoudre les problèmes éducatifs et d'inégalité sociale en milieu rural. Les enfants alternent entre enseignement théorique et travail sur l'exploitation des parents. Les agriculteurs prennent en charge les aspects financiers et pédagogiques de l'EFA en concertation avec les enseignants.

L'idée est de freiner le départ des jeunes en les préparant à la vie à la campagne et en revalorisant l'identité paysanne et régionale : « non seulement l'État ne répond pas à ses obligations, mais quand il est présent, il impose une culture, une vision. Et ce que les ruraux ont ressenti, c'est que les enfants allaient à l'école pour apprendre à détester leur propre culture, ils recevaient des enseignements qui ne leur permettaient pas de continuer à vivre ici. [...] Quand l'État ouvre une école dans cette région, c'est pour "civiliser" " (extrait d'entretien auprès d'un producteur familial et ancien élève d'une EFA, juin 2008).

En 2014, il existe environ 70 EFA en Argentine, situées majoritairement dans le Nord du pays.

* Dans le sillage du MR, deux ONG de "promotion humaine et sociale " du pays apparaissent au nord de la province de Santa Fe : INCUPO (Instituto de Cultura Popular) et FUNDAPAZ (Fundación para el Desarrollo en Justicia y Paz). Elles vont agir dans tout le Chaco en suivant sensiblement les mêmes modalités. Dans le cas d'INCUPO, si son action en faveur de l'éducation populaire est complémentaire à ses débuts de celle de l'INTA, ensuite, quand l'INTA s'oriente vers le transfert de technologies, INCUPO prend son contre-pied et propose une " pédagogie de l'autonomie du groupe ». Il promeut la diversification de la production, le renforcement de l'autoconsommation familiale et la création de " technologies adaptées » aux petits producteurs. INCUPO adapte son intervention au changement de contexte politique : à ses débuts, prime l'aspect socio-culturel à travers l'alphabétisation ; ensuite, pendant la dictature (durant laquelle les réunions collectives sont interdites ou surveillées), c'est l'aspect socio-économique et l'amélioration de la production agricole qui sont mis en avant. Avec le retour de la démocratie en 1983, renaît l'aspect politique (représentation et défense des intérêts des petits producteurs, création de plusieurs organisations représentatives).

Les techniques d'encadrement qui émergent avec le MR dès les années 1960 reposent sur un socle commun articulant dimensions éducative, sociale et culturelle, et dimensions politique et économiques. Le MR a tenté de faire le lien entre les producteurs minifundistes nés sur place et les ex- 
ploitants familiaux d'origine européenne qui représentaient l'essentiel de ses membres pour donner une autre orientation à la modernisation du secteur. Mais avec la dictature, ce lien est rompu et les conditions sont données pour une bifurcation des trajectoires agricoles. Dès lors, la distinction entre une action éducative et de développement intégral de la famille et de la communauté rurale portée par le MR, et une action de transfert dominée par une vision agronomique et technicienne préconisée par l'INTA, sépare les populations agricoles. Cette séparation va se prolonger et se traduire dans des champs d'action publique dévolus soit au développement rural, soit au développement agricole.

\section{RENOUVELLEMENT DES TECHNIQUES D'ENCADREMENT DE L'AGRICULTURE INTENSIVE ET INSTITUTIONNALISATION DE LA PETITE AGRICULTURE FAMILIALE}

Les années 1990 et la libéralisation de l'économie marquent le début d'une période de transformation forte des techniques d'encadrement de l'agriculture argentine : maintenant, c'est auprès du secteur privé que sont puisées les connaissances et les innovations, moteurs de sa croissance. $\mathrm{Pa}$ radoxalement, c'est également durant cette période que les populations pauvres, jusqu'alors considérées comme des contre-rationalités, sont rattachées à l'encadrement de l'État, via des programmes de développement rural.

\section{Objectifs de développement agricole et acteurs privés}

Alors que la décennie 1980 est synonyme d'années difficiles pour les économies latino-américaines, une phase d'amélioration de la production et de la productivité commence pour le secteur agricole argentin. La modernisation des années 1960 reprend, mais avec beaucoup plus de rapidité et de profondeur.

Les mesures macro-économiques de libéralisation adoptées à partir de 1991, dans le cadre du Consensus de Washington, bouleversent les politiques monétaire (parité fixe et surévaluation), commerciale (ouverture internationale, création du Mercosur), productive (privatisations), financière (souplesse) et sociale (flexibilisation). Tandis que l'industrie nationale s'effondre peu à peu, les mécanismes de régulation des prix et des marchés sont supprimés, ce qui dope les productions agricoles d'exportation et l'entrée de devises. L'INTA perd son autonomie en passant sous la dépendance du Ministère de l'économie, et voit son budget réduit.

La généralisation de la culture du soja est à la fois cause et effet de l'accès facilité par l'État à des intrants importés, plus performants. Les acteurs privés (banques, agro-fournisseurs) peuvent proposer des crédits bonifiés, des semences génétiquement modifiées (OGM) dont les importations et la production sont autorisées à partir du milieu des années 1990, des fertilisants, des herbicides et du matériel agricole. Le semis direct trouve sa lé- 
gitimité ; il est au centre de protocoles productifs simplifiés prônés par des organisations interprofessionnelles comme l'AAPRESID - association argentine des producteurs en semis direct, ou l'ACSOJA - association des producteurs de soja, et il polarise les actions de suivi des producteurs, menées à bien par les CREA. Ce paquet technique est à la base du paradigme agricole $^{18}$ visant à produire à moindre coût. Mais les producteurs connaissent un fort endettement auquel nombre d'entre eux ne peuvent faire face lorsque les prix varient (baisse en 1997 et 1998) ou si le coût des intrants (libellé en dollars) est renchéri. La sélection s'accélère, le nombre des producteurs passe de plus de 420000 en 1998 à un peu moins de 318000 en 2002 (recensements INDEC).

L'effondrement, fin 2001, de cette orientation macro-économique et la dévaluation du peso début 2002 va bénéficier au secteur. L’agriculture redevient compétitive. Elle fournit depuis ces années 2000 la moitié, en valeur, des exportations argentines totales. Par ailleurs, le gouvernement rétablit en 2002 les taxes sur les exportations de produits agricoles et autres biens primaires. C'est encore en 2015 le principal instrument de régulation du secteur agro-exportateur. Toutefois, si les conditions macro-économiques sont privilégiées pour évoquer le développement agricole des années 2000, la dimension technique et les innovations sont reconnues comme des facteurs décisifs : "le boom agro-exportateur n'est pas une conséquence de la dévaluation, ni des prix des commodities conjoncturellement élevés. [...] La raison majeure et fondamentale de ce moment extraordinaire que vit l'agriculture argentine n'est rien d'autre que la technologie » (discours du président de l'association des chambres de technologie agricole, 2004) ${ }^{19}$.

Les techniques d'encadrement du secteur agricole ont ainsi pour support la technique, la finance et les intérêts partagés des acteurs privés, qui sont les artisans de gains de productivité importants et de l'expansion spatiale des productions pampéennes vers les régions voisines. Le Chaco est, à ce titre, emblématique d'une attractivité qui l'entraîne sur le chemin de la « sojatisation ", autrement dit vers de nouvelles rationalités que le secteur privé définit bien plus que l'État. En effet, les terres chaquéennes jusqu'alors peu considérées, du fait de l'éloignement géographique des ports sur le Paraná (plusieurs centaines de kilomètres) et des conditions climatiques, sont maintenant convoitées par des acteurs extérieurs à la région. La rationalisation de l'espace (déforestation, drainage, irrigation) pour l'adapter aux

${ }^{18}$ R. Bisang, G. Anlló, M. Campi, « Una revolución (no tan) silenciosa. Claves para repensar el agro en Argentina », Desarrollo económico, n 48, 2008, pp. 165207.

${ }_{19} \mathrm{~J}$. Balsa, «Las disputas hegemónicas en torno de las cuestiones sociales agrarias de la Pampa argentina en la actualidad », dans M. Girbal-Blacha (dir.), Cuestiones agrarias en Argentina y Brasil, Buenos Aires, Ed. Prometeo, 2007, pp. 149-170. 
nouvelles productions (soja, élevage bovin) est inéluctable ${ }^{20}$. Les critiques concernant les impacts sociaux et environnementaux se multiplient ${ }^{21}$.

Cependant, la volonté politique « d'atténuation » des effets de l'ouverture néolibérale et l'urgence sociale dans laquelle se trouve le pays depuis les années 1990, incite l'État à mettre en place des programmes de soutien s'adressant à des publics cibles.

\section{Conformation du champ du développement rural}

Afin de faire face aux difficultés des populations rurales laissées sur le bord du chemin de la modernisation et qui subissent de plein fouet les mesures néolibérales, l'État formule des programmes de développement. Selon le recensement agricole de 2002, entre $40 \%$ et $59 \%$ des exploitations sont pauvres. Dès lors, la question de la pauvreté que l'État avait évacuée de son agenda agricole, revient habillée des valeurs du développement rural.

Ces programmes, financés par des organisations internationales (BID, FIDA, BM, PNUD), sont exécutés par les organismes publics du développement agricole, le secrétariat de l'Agriculture et l'INTA. Ce dernier prend donc en charge un nouveau type de public qui ne relevait plus de ses activités d'extensión depuis les années 1970. Ceci induit, encore aujourd'hui, des tensions internes et idéologiques au sein de l'institut. Les services de l'État, qui ont peu d'expérience dans ce domaine, font appel aux cadres cognitifs et normatifs des techniciens des ONG du Chaco qui ont développé des compétences d'intervention auprès des petits producteurs.

Les programmes visent des cibles et des objectifs différents, et déploient pour chacun d'eux des instruments propres. Parmi les plus représentatifs se trouvent:

- Le Programa Social Agropecuario (PSA). Reprenant les méthodes d'INCUPO, le PSA favorise l'organisation des producteurs et va jouer un rôle essentiel dans la structuration des objets et des cibles du développement rural, et dans la construction d'une identité propre aux acteurs et aux techniciens impliqués. Il a pour but la réinsertion des petits producteurs dans le marché et l'amélioration de leurs conditions de vie et de production (crédits, subventions, assistance technique).

${ }^{20}$ M. Gisclard, Le développement rural en Argentine, transformations de l'action publique et recompositions territoriales. Une étude à partir du Chaco argentin, Thèse de doctorat en géographie, Université de Toulouse, 2011.

${ }^{21}$ S. Federovski, "La soja como problema », Le Monde Diplomatique Edición Cono Sur, pp. 8-10, 2014.

P. Lapegna, «The Expansion of Transgenic Soybeans and the Killing of Indigenous Peasants in Argentina ", Societies Without Borders, n 8, 2, pp. 291-308, 2013.

C. Reboratti, "Le soja et l'Argentine », Problèmes d'Amérique latine, n 70, pp. 8099, 2008. 
- Cambio Rural, exécuté par l'INTA, a une fonction palliative pour les " entreprises agricoles moyennes » affaiblies par la libéralisation. Il se focalise sur leur compétitivité en les aidant, à travers du conseil, à s'adapter aux exigences des marchés ou à se reconvertir.

- Prohuerta, conduit par le ministère du Développement social et mis en œuvre par l'INTA, cherche à améliorer le régime alimentaire des familles pauvres, rurales et urbaines, en renforçant leurs capacités à produire une partie de leur alimentation selon les critères de l'agriculture biologique et grâce à des poulaillers ou des potagers familiaux.

Au regard des programmes mis en place et contrairement à une idée largement répandue, les années 1990 ne sont pas seulement les années de désengagement de l'État vis-à-vis du secteur agricole. Un autre fait majeur s'est produit en parallèle : les agriculteurs en grande partie exclus depuis les années 1970 de son encadrement, réintègrent le champ d'action sociale qu'il conduit.

\section{LA RECONNAISSANCE DE RATIONALITÉS TECHNIQUES ANTAGONISTES (2000- 2010)}

En 2009, le champ du développement rural est bouleversé lorsque la Présidente Cristina Kirchner inaugure le secrétariat de l'Agriculture familiale et du Développement rural, au sein d'un ministère, nouveau, de l'Agriculture. Nonobstant, les politiques publiques dédiées à l'agriculture familiale ne marquent pas de rupture majeure avec les programmes des années 1990. Les multiples groupes et organisations de petits producteurs éparpillés dans le territoire bénéficient toutefois d'une représentation nationale et des nouvelles prestations (sécurité sociale, retraite) ${ }^{22}$.

Si l'INTA avait déjà donné une visibilité à l'agriculture familiale comme catégorie sociale et productive en créant en 2005 des centres de recherches qui lui soient spécifiquement consacrés (CIPAF), il lui confère, également, à ce moment-là, une catégorie politique. Plusieurs processus et évènements ont conduit à cette institutionnalisation ${ }^{23}$. L'un d'eux est révélateur de la relation qu'entretient le gouvernement avec le secteur agricole. Il s'agit de la crise politique liée au conflit qui a opposé, en 2008, les syndicats agricoles au gouvernement. Suite à son annonce de transformer le système de taxes fixes sur les exportations en un système de taxes indexées sur la fluctuation des prix internationaux, les producteurs entament une " grève agri-

${ }^{22}$ La présidente améliore aussi les conditions de travail des ouvriers agricoles en 2011 grâce à une loi ( $\mathrm{N}^{\circ}$ 26.727) qui réduit le temps de travail journalier et avance l'âge de la retraite en particulier. Toutefois, le taux d'employés non déclarés dans le secteur est estimé à environ $75 \%$ par le ministère du travail (Página 12, 28.07.2010).

${ }^{23}$ M. Gisclard, G. Allaire, "L'institutionnalisation de l'agriculture familiale en Argentine : vers la reformulation d'un référentiel de développement rural ", Autrepart, $\mathrm{n}^{\circ}$ 62, pp. 201-216, 2012. 
cole » et installent des barrages routiers dans tout le pays. Beaucoup se déclarent "auto-convocados ${ }^{24}$ ", liés à aucune organisation ou parti politique.

Indirectement, ce conflit a des implications sur le champ du développement rural. Sa médiatisation et le regroupement des organisations de producteurs qui négocient collectivement leurs intérêts avec le gouvernement, donnent l'impression d'un monde agricole monolithique, constitué de producteurs exportateurs de soja. Les acteurs du développement rural s'insèrent dans le débat et donnent à voir, à une société urbaine favorable aux producteurs en grève, ce qu'ils nomment " l'autre agriculture », celle des petits exploitants. Le gouvernement joue sur cette vision dichotomique de la réalité et fait des " petits producteurs " ses alliés. Il accuse les acteurs des filières pampéennes tournées vers le marché mondial de ne pas être solidaires des populations bénéficiaires de la politique sociale financée en grande partie par les taxes sur les exportations. Si le projet de loi est finalement rejeté, le conflit accélère toutefois la création du secrétariat de l'Agriculture familiale et du développement rural, qui, par ailleurs, était vivement attendu. Au-delà de l'intérêt politique immédiat pour le gouvernement, cette nouvelle instance répond à une reconnaissance au niveau régional. Le Brésil, qui a deux Ministères de l'agriculture, a fait émerger la catégorie « agriculture familiale » dans les pays du MERCOSUR qui en retient la définition suivante : " un type de production où l'unité domestique et l'unité productive sont physiquement intégrées, l'agriculture est la principale occupation et source de revenu de la cellule familiale, la famille apporte l'essentiel de la force de travail utilisée sur l'exploitation, et la production est conjointement orientée vers l'autoconsommation et le marché ${ }^{25}$ (REAF, 2006).

Cette institutionnalisation traduit finalement la reconnaissance politique des techniques d'encadrement issues des années 1970. Elle se produit en effet dans un contexte politique qui vise la "récupération » de l'héritage politique de ces années-là et la " réparation » aux victimes de la dictature (leaders paysans et ensemble des exclus des politiques publiques officielles) et aux régions restées en marge de la croissance économique pampéenne.

Par ailleurs, la création du secrétariat de l'agriculture familiale, dans un contexte conflictuel, situe cette catégorie en contre-pied de l'agriculture entrepreneuriale et vient souligner la logique émancipatrice sous-jacente aux critiques formulées par l'agriculture familiale au moment du conflit de $2008^{26}$. Chacune a désormais des missions différentes, légitimées politiquement au sein de la société et du territoire. D'un côté, l'agriculture familiale, qui produirait entre $25 \%$ y $30 \%$ des biens agricoles du pays, doit assurer

${ }^{24}$ Cette volonté de se démarquer des partis politiques traditionnels est, selon nous, un signe supplémentaire de la déconnexion du secteur et de l'État.

${ }^{25}$ REAF, Reunión especializada sobre Agricultura Familiar, FIDA Mercosur, Brasilia, 2006.

${ }^{26}$ M. Gisclard, F. Goulet, « Analyser les processus de catégorisation de l'agriculture familiale en Argentine », Colloque Les Petites paysanneries face à un contexte mondial incertain, Université Paris Ouest, Nanterre, 19-21 novembre 2014. 
la sécurité alimentaire nationale. De par ses pratiques et modes de culture, elle est perçue comme agissant favorablement pour la protection de l'environnement (moindre dégradation des ressources productives). Son soutien s'inscrit dans une stratégie de lutte contre la pauvreté : $66 \%$ des exploitations agricoles sont familiales (dont $55 \%$ qui produisent pour leur seule subsistance). Employant $53 \%$ de la main d'œuvre agricole, soit 428157 postes de travail ${ }^{27}$, elles garantissent le maintien d'un tissu social local dans un pays où la population rurale ne représente plus que $12,5 \%$ de la population totale, et où la population rurale économiquement active ne compte que pour $8 \%$ environ de la population active totale. Les exploitations de petits producteurs travaillent encore 23,5 millions d'ha, soit 13,5\% de la superficie agricole de l'Argentine. En synthèse, la catégorie politique de l'agriculture familiale est investie d'une mission à la fois productive, sociale et environnementale.

De l'autre, les acteurs de l'agriculture entrepreneuriale, moteurs depuis 2002 d'un certain dynamisme économique, abondent une balance commerciale composée de biens primaires ou semi-transformés. La politique de redistribution sociale vers les secteurs les plus vulnérables (dont les petits agriculteurs familiaux) dépend par ailleurs des taxes aux exportations qui rapportent environ 10 milliards de dollars/an. Les agricultures entrepreneuriales, dans leur diversité et leurs logiques, sont donc le nœud central d'une logique d'accumulation basée sur l'exportation de matières premières, logique qualifiée de $"$ consensus des commodities ${ }^{28}$. Ce terme souligne le maintien de la logique agro-exportatrice néolibérale malgré le discours du gouvernement s'inscrivant en rupture avec elle. Le Plan Stratégique Agricole et Agro-alimentaire (PEA2) établit, à l'horizon 2020, une augmentation de $60 \%$ de la production de céréales et oléo-protéagineux et de $40 \%$ de l'élevage. Ces objectifs ne pourront être remplis qu'au prix d'une augmentation de la productivité et de la mise en culture de nouvelles terres.

Le secteur agricole intensif continue ainsi à se détacher des structures d'encadrement de l'État pour créer ses propres systèmes d'innovation pilotés par les acteurs privés. Les producteurs familiaux capitalisés et les entreprises agricoles s'inscrivent désormais dans des faisceaux démultipliés de relations avec les acteurs de l'agrofourniture et de l'agro-industrie. Les acteurs en amont et en aval, coopératives, grands opérateurs internationaux de la production et de la diffusion de semences, de matériel ou d'autres innovations, entreprises agro-industrielles étrangères et nationales à la pointe, sont proches des producteurs qu'ils conseillent et à qui ils ven-

${ }^{27}$ P. Juarez, M. Gisclard, F. Goulet, A. Albaladejo, J. Elverdin, R. Cittadini, M. Patrouilleau, Políticas públicas y desarrollo rural en América latina: el caso de la agricultura familiar en la República argentina », dans CEPAL, Políticas públicas y agriculturas familiares en América latina: situación, desafíos y perspectivas, Santiago de Chile, 2014.

${ }^{28}$ M. Svampa, " “Consenso de los Commodities” y lenguajes de valoración en América Latina » Nueva Sociedad, n² 244, pp. 29-46, 2013. 
dent leurs innovations. Ils leur achètent des biens agricoles valorisés lors des étapes de transformation agro-industrielle et selon les exigences du marché.

Les innovations sont aussi organisationnelles dans la mesure où la recherche d'efficacité productive et d'économies d'échelle pousse à travailler des parcelles agrandies, et à optimiser l'utilisation du matériel agricole et du foncier, quitte à ne pas l'acquérir : la sous-traitance des travaux agricoles et la location de terres sont prédominantes. Les contratistas, entrepreneurs de services agricoles équipés en machines dernière génération, diffusent les ultimes innovations en matière de semis, traitement et récolte ; les cabinets d'ingénieurs agronomes conseillent à titre privé autant que les semenciers et agro-fournisseurs. Les filières d'exportation sont animées aussi par des entreprises de production agricole d'envergure (milliers d'hectares) et dont l'organisation repose sur une gestion scrupuleuse des facteurs de production $^{29}$, dépendante des retours sur investissement attendus par les actionnaires. L'agriculture entrepreneuriale argentine fait donc preuve de grande diversité et est en quête d'innovations, d'investissements et de rentabilité à court terme. Le tout sur fond d'offre accrue de moyens de communication et de transmission des informations, et de techniques de commercialisation (outils boursiers, logistique, etc.).

Á la recherche de foncier, l’agriculture entrepreneuriale est la principale responsable de l'avancée de la frontière dans des régions périphériques comme le Chaco. Les conséquences sont l'objet de critiques provenant de la société civile et des organisations de l'agriculture familiale : illégalité et massivité de la déforestation (1 250000 ha défrichés dans le nord du pays entre 1998 y $2006^{30}$ ), pollution des eaux et des sols avec l'augmentation de l'usage des pesticides et des fertilisants (respectivement, hausse de $200 \%$ et de $100 \%$ entre 1998 et $2011^{31}$ ), santé des populations rurales, etc.

Si les conditions et les conséquences de l'avancée de la frontière agricole reposent sur un terreau institutionnel hérité du passé, la pression foncière, l'expulsion de petits producteurs et l'avancée sur les territoires indigènes (plusieurs assassinats de leaders paysans ces dernières années) sont rendus possibles par la faiblesse des organismes de contrôle et la corruption des élites politiques locales.

${ }^{29}$ M. Guibert, S. Grosso, P. Arbeletche, M. E. Bellini, « De Argentina a Uruguay: espacios y actores en una nueva lógica de producción agrícola », Revista Pampa, Suplemento especial, $\mathrm{n}^{\circ}$ 7, pp. 13-38, 2011.

M. Guibert, « Les formes associatives de production agricole en Argentine et en Uruguay : entre territoire et réseau ? », Études rurales, n 191, pp. 77-90, 2013.

${ }^{30}$ SAyDS - Secretaría de Ambiente y Desarrollo Sustentable de la Nación, 2012. Monitoreo de la Superficie de Bosque Nativo de la República Argentina. Período 20062011. Regiones forestales Parque Chaqueño, Selva Misionera y Selva Tucumano Boliviana. http://www.ambiente.gov.ar/archivos/web/UMSEF/file/LeyBN/monitoreo _bn_2006_2011_ley26331.pdf

${ }^{31}$ S. Federovski, "La soja como problema », Le Monde Diplomatique Edición Cono Sur, pp. 8-10, 2014. 
Malgré l'institutionnalisation de l'agriculture familiale, et la mise en place d'un nouveau cadre législatif, l'État ne régule que marginalement ces phénomènes. Depuis 2007, la loi de protection des forêts est censée freiner la déforestation. Les difficultés de son application renvoient à la connivence entre agriculteurs entrepreneuriaux et pouvoirs publics ${ }^{32}$, à la faiblesse des compétences techniques environnementales ${ }^{33}$ et des capacités de contrôle. Autre exemple : la concentration de la terre n'est abordée par le gouvernement qu'à travers la loi de 2009 de régulation de l'achat de terres par des acteurs étrangers. Elle fixe le seuil maximal de terres possédées par des étrangers à $20 \%$ d'une superficie provinciale et à $15 \%$ de la superficie nationale. Or, selon une enquête publique nationale, seulement $6 \%$ de la terre est entre des mains étrangères. La loi s'inscrit donc dans une stratégie rhétorique et symbolique du gouvernement qui modifie très peu les phénomènes à l'œuvre.

\section{CONCLUSION}

L'institutionnalisation de l'agriculture familiale est un fait récent en Argentine qui intervient en contre-point de la consolidation d'un modèle d'agriculture entrepreneuriale hérité de décennies de libéralisation progressive du secteur et enraciné dans la configuration historique du pays. Or, si l'économie approfondit le modèle lié à l'exportation de biens primaires, la nécessité d'assurer la sécurité alimentaire nationale et de soutenir les populations rurales pauvres (re)donnent aux petits agriculteurs une légitimité et provoquent un regain d'attention de la part des autorités qui peuvent sembler paradoxaux dans le contexte argentin. Loin de considérer cette coexistence comme évidente, nous avons voulu comprendre les origines de l'éclatement du secteur agricole en suivant les trajectoires sociohistoriques respectives de ces modèles de développement jusqu'à leur actuelle légitimation politique.

Nous avons montré tout d'abord que leurs trajectoires se séparent au moment de la modernisation agricole portée par l'État. Les techniques d'encadrement qu'il promeut dans un cadre macro-économique qui se libéralise, excluent les populations rurales pauvres qui ne correspondent pas à l'archétype du producteur moderne et dynamique. Du coup, elles développent leurs propres techniques d'encadrement qui renvoient à une problématique sociale. Le développement rural et le développement agricole qui émergent comme des champs d'action publique dans les années 1990, reposent ainsi sur la séparation des techniques d'encadrement des années 1960 : d'une part, l'action d'éducation et de développement intégral de la communauté rurale portées par le Movimiento Rural, et, d'autre part, l'ac-

${ }^{32}$ F. Silvetti, G. Soto, D. Cáceres, D. Cabrol, « ¿Por qué la legislación no protege los bosques nativos de Argentina? Conflictos socio-ambientales y políticas públicas», Mundo agrario, vol. 13, $\mathrm{n}^{\circ} 26,2013$.

${ }^{33}$ L.E. Ruoso, Stratégies de protection de la forêt et production de savoirs environnementaux, Mémoire de Master en géographie, Université Paris 1, 2012. 
tion transfériste dominée par une vision agronomique et technicienne, portée par l'INTA en particulier.

Ensuite, l'analyse démystifie le " tournant néolibéral » des années 1990 et ses effets sur le secteur agricole. S'il a apporté les conditions macro-économiques pour que la technique devienne un des principaux médiateurs de la structuration du secteur agricole et de l'agriculture entrepreneuriale, les conditions institutionnelles de cette bifurcation peuvent être observées dans l'évolution de la régulation du secteur par l'État. Sans nier l'ampleur des effets de la politique de libéralisation, la pauvreté rurale n'est pas le produit de cette seule ouverture économique, elle est également issue d'un fond historique et institutionnel complexe, et d'une modernisation agricole sélective. De même, les " techniques d'encadrement » actuelles des agricultures technicisées, basées sur l'adoption de technologies de plus en plus innovantes et performantes en termes de rentabilité, proviennent également de ce terreau socio-institutionnel, déterminé dès les années 1970. Ainsi, l'agriculture entrepreneuriale, aujourd'hui la plus détachée des structures d'encadrement de l'État, est pourtant le résultat des modalités de son action de modernisation. De même, les difficultés rencontrées par l'agriculture familiale (aujourd'hui reconnue par l'Etat) sont le résultat d'années de non planification de son devenir.

En définitive, la région du Chaco illustre les difficultés à réguler les effets sociaux et environnementaux de l'agriculture entrepreneuriale qui remet en question le maintien d'une agriculture paysanne et familiale pourtant amenée à jouer un rôle important dans l'approvisionnement des marchés nationaux. Les techniques d'encadrement actuelles des différents modèles agricoles ne prennent pas en compte les tensions liées à leur cohabitation dans les mêmes espaces. Ceci témoigne de l'hégémonie du référentiel productiviste agro-exportateur et aussi des difficultés du gouvernement de Cristina Kirchner de construire un projet national duquel aurait émergé un modèle clair de développement et de régulation. Tout se passe comme si le référentiel social et environnemental associé à la petite agriculture familiale n'était qu'un moyen de faire accepter le référentiel productif dominant.

Ainsi, contrairement à ce que la reconnaissance politique des agricultures familiales pourrait laisser penser, celles-ci font l'objet de techniques d'encadrement qui ne marquent pas de rupture nette avec les politiques précédentes. Jusqu'à présent, l'absence de mesures fortes pour contrer les véritables problèmes structurels des petits producteurs (tenure de la terre, formation, accès aux marchés, infrastructures de transport dans les régions périphériques, etc.) pourrait laisser penser que la petite agriculture familiale a été l'objet plus d'une stratégie politique de la part du gouvernement que d'un véritable programme de développement établi à long terme. Le travail de légitimation politique de ce secteur par le gouvernement et l'empowerment des organisations de petits producteurs qu'il appelait de ses vœux, sont maintenant mis à l'épreuve de l'alternance politique entamée en décembre 2015 qui a amené au pouvoir un gouvernement de centredroit, plus prompt à défendre et à favoriser l'agriculture entrepreneuriale. 